\title{
Estudio del síndrome de burnout en deportistas: prevalencia y relación con el esquema corporal*
}

\author{
A Study of the Burnout Syndrome in Sportspeople: \\ Prevalence and Relationship to the Body Image
}

Recibido: octubre 29 de 2012 | Revisado: abril 10 de 2013 | Aceptado: abril 10 de 2013

\author{
IGNACIO PEDROSA ** \\ EDUARDO GARCÍA-CUETO \\ Universidad de Oviedo, España
}

doi:10.11144/Javeriana.UPSY13-1.esbd

Para citar este artículo: Pedrosa, I. \& García-Cueto, E. (2014). Estudio del síndrome de burnout en deportistas: prevalencia y relación con el esquema corporal. Universitas Psychologica, 13(1), 135-143. doi:10.11144/Javeriana.UPSY13-1.esbd

* Investigación financiada por el Programa de Formación de Personal Universitario del Ministerio de Educación del Gobierno de España (AP2010-1999).

** Facultad de Psicología. E-mails: npedrosa@cop.es, cueto@uniovi.es
RESUMEN

Recientemente, se han descubierto diferentes variables psicológicas tanto generadoras como potenciadoras del síndrome de burnout. A esto, hay que añadir la alta prevalencia del síndrome en deportistas a nivel internacional. El objetivo del trabajo ha sido establecer la prevalencia del síndrome en población deportista española, así como analizar su relación con la percepción del esquema corporal. En el estudio han participado 397 deportistas (59.8\% hombres) de entre 13 y 64 años $(M=19.23$ y $D E=6.67)$ de diferentes modalidades deportivas (35\% deportes individuales). Los resultados muestran una prevalencia del síndrome de burnout en el contexto deportivo nacional de $2.77 \%$, así como la existencia de una relación entre el síndrome y una percepción negativa del esquema corporal, especialmente con las dimensiones de devaluación $\left(r_{\mathrm{xy}}=0.336\right)$ y realización personal $\left(r_{\mathrm{xy}}=\right.$ 0.44). Además, mediante ecuaciones estructurales, la percepción corporal negativa puede considerarse una consecuencia psicológica añadida a las derivadas del propio síndrome.

Palabras clave autores

Síndrome de burnout, deportistas, prevalencia, esquema corporal.

Palabras clave descriptores

Psicología de la salud, investigación cuantitativa, España.

\section{A B S T R A C T}

Different psychological variables have recently been discovered as enablers of the burnout syndrome. Moreover, it must be added the high prevalence rates of the syndrome on international sportspeople. The aim of the paper was to establish the prevalence rate of the syndrome in the Spanish sportspeople and to analyze its relationship with the perception of body image. 397 sportspeople took part in the research (59.8\% men) aged between 13 and 64 years old $(M=19.23$ and $S D=6.67)$ of different sports $(35 \%$ individual sports). The results show a prevalence rate of burnout syndrome in the context of national sports $2.77 \%$ and the existence of a relationship between the syndrome and a negative body image perception, especially with the dimensions of devaluation $\left(r_{x y}=0.336\right)$ and reduced sense of accomplishment $\left(r_{\mathrm{xy}}=0.44\right)$. Moreover, using SEM, negative body image could be proposed as a psychological consequence added to those derived from the syndrome itself.

Keywords authors

Burnout syndrome, sportspeople, prevalence, body image.

Keywords plus

Health Psychology, Quantitative Research, Spain. 
El síndrome de burnout es un trastorno que afecta a profesionales de diversas áreas como los docentes, el personal sanitario o los trabajadores del ámbito social (Bernaldo de Quirós-Aragón \& LabradorEncinas, 2007; Díaz, López \& Varela, 2012; JenaroRío, Flores-Robaina \& González-Gil, 2007; LeónRubio, Cantero \& León-Pérez, 2011) y entre los que también se encuentran los deportistas.

El síndrome de burnout, en el contexto deportivo, se define como la presencia de un agotamiento físico y emocional (A) originado por unas exigencias elevadas en el entorno competitivo, una baja realización personal (RP), debida a una falta de consecución de los sentimientos de éxito y de crecimiento individual en el contexto deportivo, y una devaluación del deporte (D) que causaría una pérdida de interés por la propia actividad (Raedeke, 1997).

Si bien los primeros trabajos en relación con este síndrome se ocuparon de establecer tanto los aspectos psicológicos y sociales implicados en la aparición del síndrome de burnout (Etzion, 1984; Feigley, 1984) como en la elaboración de los primeros modelos explicativos (Capel, 1986; Smith, 1986), actualmente, los diversos trabajos tratan de descifrar las variables psicológicas causantes de su aparición y desarrollo. De hecho, desde el establecimiento del modelo tridimensional de Raedeke (1997), la investigación en el contexto deportivo ha crecido progresivamente, aumentando de manera significativa en los últimos años (Pedrosa \& GarcíaCueto, 2010). Este incremento sobre el burnout en deportistas se debe a dos causas fundamentales: la elevada prevalencia que presenta y las importantes consecuencias, tanto individuales como sociales, que provoca.

Respecto al primer aspecto, diferentes estudios han demostrado la alta prevalencia que tiene este trastorno en la población deportiva española, situándolo entre el 7.62 y el $8.69 \%$ (Garcés de Los Fayos, 1999; Vives Benedicto \& Garcés de los Fayos, 2004). El problema es que estos datos han sido obtenidos con un instrumento de evaluación basado en la concepción clínica del burnout aportada por Maslach y, por lo tanto, obvia aspectos implícitos al contexto deportivo que deben ser tenidos en cuenta, como por ejemplo, la modificación de la dimensión de despersonalización por la devaluación del propio deporte. Este aspecto de la especificidad deportiva, sí ha sido tenido en cuenta por otros trabajos a nivel internacional, los cuales cifran el burnout entre el 1 y el 5\% de manera general (Gustafsson, Kenttä \& Hassmén, 2011; Gustafsson, Kenttä, Hassmén \& Lundqvist, 2007), mientras que por sexos, este se cifra de un 2 a un 6\% para los varones y un 1 a un $9 \%$ para las mujeres (Gustafsson et al., 2007).

En cuanto a sus consecuencias, se ha demostrado que el síndrome de burnout adquiere una alta relevancia por presentar repercusiones significativas a nivel afectivo, cognitivo, motivacional y comportamental (Goodger, Gorely, Lavallee \& Harwood, 2007; Gustafsson, Hassmén, Kenttä \& Johansson, 2008). De hecho, se ha confirmado que afecta al rendimiento del deportista y que, incluso mantenido en el tiempo, puede ocasionar el abandono de la práctica deportiva (Carlin \& de los Fayos Ruiz, 2010; Gustafsson et al., 2008).

Como se ha expuesto, recientemente se han postulado diferentes variables psicológicas que han demostrado presentar un efecto importante tanto sobre la generación como sobre el desarrollo del síndrome de burnout en el ámbito deportivo. En este sentido, existen variables que han indicado su influencia de manera sistemática como la ansiedad, el apoyo social, el estrés o la depresión, esta última entendida por la influencia que tiene tanto por sî misma como por ser variable mediadora, como se expone más adelante, entre otras (Austria, Cruz, Herrera \& Salas, 2012; Black \& Smith, 2007; Conde et al., 2010; Cresswell \& Eklund, 2004; González, Molinero \& Márquez, 2008).

Sin embargo, a ellas se han sumado recientemente factores personales como el optimismo (Gustafsson \& Skoog, 2012), el cual afecta, mediante el estrés percibido, especialmente a las dimensiones de agotamiento físico y emocional y devaluación del deporte; la resistencia a la frustración (Gustafsson, Hassmen \& Podlog, 2010); o el propio exceso de entusiasmo, cuando se interpreta como un exceso de práctica deportiva, bien en entrenamiento o competición, así como una falta de recuperación adecuada (Gustafsson, 2011). 
Resulta fundamental, por tanto, continuar investigando qué variables pueden estar ligadas al síndrome con el objetivo de conocer así todas las que están implicadas en el mismo y poder, de esta manera, llevar a cabo una prevención e intervención mucho más eficaz.

En este sentido, teniendo en cuenta las investigaciones existentes, el estrés percibido por el deportista destaca como variable, tanto causante como mediadora, del síndrome de burnout. Aunque a día de hoy no se ha realizado ningún estudio que haya relacionado la percepción del esquema o la imagen corporal, entendida tanto en relación con las dimensiones, forma y componentes del cuerpo, como con las capacidades para el movimiento del propio cuerpo del deportista y sus interacciones con el ambiente (Cratty, 1982), con el propio síndrome, síque se puede afirmar la relación entre el estrés y la percepción del esquema corporal $\left(r_{\mathrm{xy}}=0.613\right)$ (Pedrosa, Suárez-Álvarez \& García-Cueto, 2012). Así, es presumible pensar que un deportista con una percepción negativa de sí mismo, no se sienta completamente capacitado para afrontar una exigencia externa.

A esto hay que sumar la existencia de trabajos que demuestran que se trata de una variable de riesgo, puesto que, como concluyen Petrie, Greenleaf, Carter y Reel (2007) en un trabajo con 199 deportistas universitarios, en casos extremos y bajo determinadas condiciones estresoras y ambientales, es posible que el deportista desarrolle un trastorno de la conducta alimentaria. Bajo este razonamiento, otros estudios similares confirman este efecto de los estresores competitivos sobre la aparición de trastornos alimentarios (Baum, 2006; HolmDenoma, Scaringi, Gordon, Van Orden \& Joiner, 2009; Sundgot-Borgen \& Torstveit, 2010).

Por tanto, teniendo en cuenta dichos estudios y, a su vez, asumiendo tanto la influencia del estrés como la relación confirmada en el ámbito clínico entre la percepción del esquema corporal y el desarrollo de trastornos alimentarios (Ferreira, Pinto-Gouveia \& Duarte, 2011; Lobera et al., 2009; Murray, Byrne \& Rieger, 2011), parece coherente plantear la existencia de una relación entre el síndrome de burnout, asumiendo el propio estrés como variable mediadora, y su efecto sobre la percepción del esquema corporal en el contexto del deporte.

Los objetivos propuestos en el presente trabajo son, en primer lugar, establecer la prevalencia actual del síndrome de burnout en deportistas españoles mediante un instrumento de medida específico del contexto deportivo y, por otra parte, analizar la posible relación del síndrome respecto a la percepción del esquema corporal que presenta el deportista, entendiendo esta variable como otro aspecto negativo adicional relacionado con el síndrome, que puede afectar notablemente el rendimiento y el bienestar psicológico del deportista. Este segundo objetivo se entiende como totalmente novedoso, al no haber sido estudiado específicamente en la literatura existente a día de hoy a nivel internacional.

\section{Método}

\section{Participantes}

Para la selección de los participantes se llevó a cabo un muestreo estratificado de diferentes clubes deportivos españoles, en función de su ubicación geográfica y la modalidad deportiva evaluada con la finalidad de alcanzar la mayor representatividad posible del panorama deportivo nacional. Con base en el mismo, en el estudio participaron 397 deportistas federados de distintos puntos del territorio nacional que competían de manera regular $(59.8 \%$ hombres y $40.2 \%$ mujeres). En concreto, los datos fueron recabados de las Comunidades Autónomas del Principado de Asturias, Galicia, Cataluña y las Islas Canarias.

El rango de edades se situó entre los 13 y los 64 años $(M=19.23$ y $D E=6.67)$, siendo la mayor parte de los deportistas evaluados jóvenes entre 13 y 30 años. A la hora del estudio de la prevalencia, se decidió dividir las edades en dos grandes grupos: adolescentes, en donde se incluyeron a todos los deportistas hasta los 18 años (55.8\% de la muestra) y adultos, entendidos como aquellos atletas con edades superiores al grupo precedente $(44.2 \%$ de la muestra). Esta diferenciación se debe a que, generalmente, las exigencias deportivas se incrementan en torno a los 18 años, pasando de un período de formación a otro de competición. 
Además, se ha tenido en cuenta la inclusión de deportes tanto individuales (35\%) como grupales (65\%), valorando los siguientes deportes: fútbol, baloncesto, balonmano, voleibol, piragüismo, artes marciales y natación. Por otro lado, los atletas evaluados presentan diferentes niveles de competición, así un 63.4\% disputa competiciones de ámbito regional; el 34.3\% compite a nivel nacional y, finalmente, un $2.3 \%$ participa en competiciones internacionales como Campeonatos de Europa o del Mundo.

\section{Instrumentos}

El cuestionario empleado en la evaluación fue la adaptación al castellano del Athlete Burnout Questionnaire (ABQ), el cual consta de 15 ítems en una escala tipo Likert de 5 alternativas y ha demostrado unas propiedades psicométricas adecuadas en cuanto a su validez y fiabilidad (Pedrosa \& GarcíaCueto, 2012).

Por otra parte, se decidió incluir la Escala de Percepción del Esquema Corporal (Pedrosa, MartínVázquez \& García-Cueto, 2008), que consta de 30 ítems en una escala tipo Likert de 5 alternativas y presenta una fiabilidad, estimada mediante el coeficiente $\alpha$ de Cronbach, de 0.96 .

\section{Procedimiento}

La aplicación de ambas escalas se realizó en formato de papel y lápiz, de manera voluntaria y colectiva, en un lugar habilitado para tal efecto dentro de las instalaciones deportivas, bajo unas instrucciones pautadas presentes en la hoja de respuestas para evitar el sesgo del aplicador. En todo momento, se aseguró el anonimato y la confidencialidad de las respuestas. Además, se obvió el uso del término burnout por las connotaciones negativas que pudiese generar en los participantes.

\section{Análisis de los datos}

En primer lugar, se realizó un estudio de la prevalencia del síndrome dentro del panorama deportivo nacional. De esta manera, se calcularon los índices de prevalencia para la muestra global, así como en función de la edad, el sexo y la modalidad deportiva practicada.

A continuación, se estudiaron las propiedades psicométricas de la Escala de Percepción del Esquema Corporal en la muestra actual, estimando su fiabilidad mediante el coeficiente $\alpha$ de Cronbach, así como su validez de constructo mediante análisis factorial.

Posteriormente, se analizó la relación entre las dimensiones del síndrome de burnout y la percepción del esquema corporal mediante la correlación de Pearson. Además, se aplicó un Análisis de Ecuaciones Estructurales teniendo en cuenta ambas variables, que permitiera valorar los efectos entre las mismas y determinar si la percepción del esquema corporal se trata de una variable antecedente o consecuente del propio síndrome.

Finalmente, mediante la prueba $t$ de Student, se analizaron las diferencias existentes en la percepción del esquema corporal en función del sexo y la edad de los participantes.

\section{Resultados}

\section{Prevalencia del síndrome de burnout}

En primer lugar, se realizó un estudio de la prevalencia del síndrome a nivel nacional en función de las variables sociodemográficas de los deportistas. Para esto, se tuvieron en cuenta los criterios de Raedeke y Smith (2001) ya expuestos (Tabla 1).

Como se puede observar, la prevalencia sobre la muestra total a nivel nacional se establece entre el 0.25 y el $2.77 \%$ tomando el riesgo muy alto o alto de padecer el síndrome, respectivamente. En función de las variables sociodemográficas, el mayor riesgo es para las mujeres, los adultos y los deportistas que practican deportes de equipo, todos ellos con valores de $0.25 \%$.

\section{Propiedades psicométricas del instrumento}

A pesar de las buenas propiedades psicométricas de la Escala de Percepción del Esquema Corporal ya mostradas previamente, se estimó su fiabilidad en el presente trabajo mediante el coeficiente $\alpha$ de Cronbach, obteniendo un valor de 0.95 y donde 
TABLA 1

Estudio de las puntuaciones en las escalas del ABQ en función de edad, sexo y modalidad deportiva

\begin{tabular}{lcc}
\hline & \multicolumn{2}{c}{ Puntuación } \\
& $>3$ todas las escalas \% & $>4$ todas las escalas \% \\
\hline Muestra total & 2.77 & 0.25 \\
\hline Hombres & 1.51 & 0 \\
Mujeres & 1.26 & 0.25 \\
Adolescentes & 1.01 & 0 \\
Adultos & 1.76 & 0.25 \\
Deportes individuales & 0.76 & 0 \\
Deportes grupales & 2.02 & 0.25 \\
\hline
\end{tabular}

Fuente: elaboración propia.

todos los ítems presentaron un índice de homogeneidad superior a 0.27 .

Referente a su validez de constructo, se probó la unidimensionalidad de la escala mediante el método de extracción de mínimos cuadrados, el factor explica el 39\% de la varianza total y muestra los índices de ajuste que aparecen en la Tabla 2.

\section{Relación entre el síndrome de burnout} y la percepción del esquema corporal

Respecto al análisis de la relación entre el síndrome de burnout y la percepción del esquema corporal, se llevó a cabo una correlación de Pearson entre las puntuaciones obtenidas por los participantes en cada una de las escalas (Tabla 3).

Como se puede comprobar, existen correlaciones estadísticamente significativas $(p<0.05)$ respecto a cada una de las tres escalas que componen el síndrome, obteniendo el valor inferior en relación con la dimensión de agotamiento físico y emocional y destacando las dimensiones que cuentan con un mayor componente psicológico como son la devaluación del deporte $\left(r_{\mathrm{xy}}=0.336\right)$ y, especialmente, la baja realización personal $\left(r_{\mathrm{xy}}=0.44\right)$.

Con base en dicha relación, se aplicó un modelo de ecuaciones estructurales que permitiese comprobar la hipótesis de que el síndrome de burnout po-

TABLA 2

Índices derivados del análisis factorial en la Escala de Percepción del Esquema Corporal

\begin{tabular}{cccc}
\hline$\chi^{2} / g l$ & AGFI & KMO & RMSR \\
(C. Kelly)
\end{tabular}

Fuente: elaboración propia.

TABLA 3

Correlaciones entre las escalas del ABQ y la percepción del esquema corporal

\begin{tabular}{cc}
\hline & Per. Esq.Corp. \\
\hline A. ABQ & $0.135^{(*)}$ \\
D. ABQ & $0.336^{(* *)}$ \\
RP. ABQ & $0.44^{(* *)}$ \\
\hline
\end{tabular}

** La correlación es significativa al nivel 0.01 .

* La correlación es significante al nivel 0.05 .

Fuente: elaboración propia. 


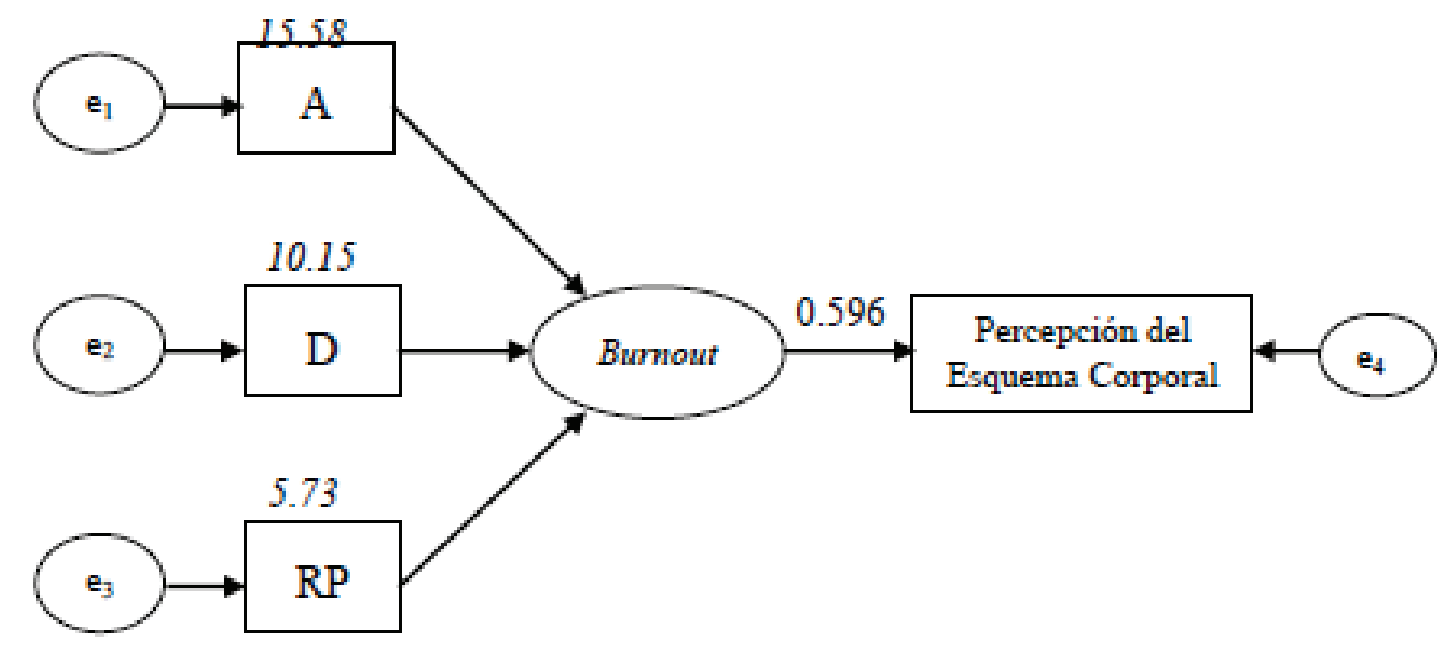

Figura 1. Modelo de ecuaciones estructurales de la relación burnout-percepción del esquema corporal.

Fuente: elaboración propia.

dría ser la causa del incremento de una percepción del esquema corporal distorsionada. En la Figura 1 se puede observar la relación estandarizada entre ambas variables y, en cursiva, el porcentaje de varianza explicado por cada una de las dimensiones dentro del síndrome de burnout. De esta manera, se puede comprobar empíricamente el efecto que el propio síndrome ejerce sobre la percepción negativa del esquema corporal.

Por otro lado, dentro del modelo, se comprueba que la dimensión de agotamiento físico y emocional (A) es la que explica el mayor porcentaje de varianza dentro del propio síndrome de burnout.

\section{Diferencias en la percepción del esquema corporal}

Finalmente, se analizaron las diferencias existentes en la percepción del esquema corporal en función del sexo y la edad, la cual se recodificó en los dos grupos descritos previamente. Además, se calcula- ron tanto la potencia de la prueba como el tamaño del efecto (d) (Tabla 4).

Como se muestra, se encontraron diferencias estadísticamente significativas en función del sexo, siendo las mujeres quienes manifestaron una peor percepción corporal. Dichas diferencias no se detectaron en función de la edad (adolescentes vs. adultos). Sin embargo, los resultados muestran una tendencia a que los deportistas adultos presenten una peor imagen de su imagen corporal. Aun no detectando diferencias estadísticamente significativas en función de la edad, tanto la potencia de la prueba como el tamaño del efecto muestran valores que se pueden considerar aceptables.

\section{Discusión y conclusiones}

El estudio de la prevalencia aporta datos actuales sobre la presencia del síndrome, el cual ha demostrado tener unas importantes consecuencias, a nivel individual y social, en España, con valores en-

\section{TABLA 4}

Estudio de diferencias en percepción del esquema corporal en función de las variables sociodemográficas

\begin{tabular}{lccc}
\hline Variable de agrupación & $p$ & Potencia & $d$ \\
\hline Sexo & $<0.001$ & 0.97 & 0.52 \\
Edad & 0.085 & 0.41 & 0.24 \\
\hline
\end{tabular}

Fuente: elaboración propia 
tre el 0.25 y el $2.77 \%$. Estos datos concuerdan con los obtenidos a nivel internacional (Gustafsson et al., 2007) y confirman así la tendencia existente en otros países analizados. En España, Vives Benedicto y Garcés de Los Fayos (2004) encuentran valores muy superiores a los aquí reseñados; tal vez debido a que en sus investigaciones no utilizan cuestionarios propios del mundo deportivo, sino escalas clínicas adaptadas al deporte. Cabe destacar que los datos de la muestra actual, no solo han sido hallados mediante un cuestionario diseñado específicamente para el contexto deportivo, sino que implica el uso del mismo instrumento de evaluación, obviamente adaptado al idioma de aplicación, que ha sido utilizado en los estudios donde se aportan dichos datos de prevalencia, lo cual fortalece aún más la lógica de obtener valores similares a los aportados a nivel internacional por Gustafsson et al. (2007) y Gustafsson et al. (2011) permitiendo, además, establecer una equivalencia mucho más veraz debido a que, como es sabido, dentro de los modelos de teoría clásica de los test, la medición de la variable no es independiente del instrumento utilizado.

Dentro de este apartado, comparando los resultados de la muestra española respecto a los obtenidos en la versión original (Raedeke $\&$ Smith, 2001), se puede destacar la existencia de patrones invertidos en relación con el síndrome en función de la edad y la modalidad deportiva. Así, mientras en la versión original los mayores índices aparecen en deportes individuales y adolescentes, en el caso de la adaptación española, las proporciones más altas se dan en deportistas adultos y en aquellos que practican deportes de equipo. Este resultado parece sensato, puesto que en estos últimos los deportistas tienen un menor margen de influencia sobre los resultados y éxitos por alcanzar y, por otro lado, la mayor exigencia deportiva comienza a aparecer a nivel profesional, es decir, cuando abandonan la adolescencia.

Respecto al instrumento empleado para valorar la percepción del esquema o la imagen corporal, en el presente estudio, se reafirman las adecuadas propiedades psicométricas del mismo obtenidas en trabajos precedentes (Pedrosa, Martín-Vázquez \&
García-Cueto, 2008), tanto a nivel de fiabilidad como de validez de constructo.

En cuanto a la variable de percepción del esquema corporal, destaca la correlación lineal positiva en relación con las tres escalas del ABQ que parece implicar un problema psicológico añadido a los ya conocidos y relacionados con el síndrome.

Hasta el momento, esta variable no había sido tenida en cuenta respecto al síndrome pero, con base en los resultados obtenidos, parece sensato plantear la existencia de una importante relación positiva entre esta y las dimensiones $\mathrm{D}$ y RP del $\mathrm{ABQ}$, destacando cómo altos niveles de devaluación del deporte, unidos a una baja realización personal, se relacionan con una percepción negativa del esquema corporal.

Asimismo, no solo se puede destacar la confirmación de esta relación, sino que se considera relevante el hecho de aplicar un modelo de ecuaciones estructurales puesto que, en vista de los datos obtenidos, parece aceptable entender las alteraciones de la percepción del esquema corporal como una consecuencia negativa que debe añadirse a las alteraciones cognitivas, emocionales, sociales y comportamentales ya conocidas derivadas del síndrome de burnout. Además, hay que recordar que, como muestran estudios como los de Petrie et al. (2007), alteraciones de esta variable, en casos extremos y bajo determinadas condiciones estresoras y ambientales, pueden provocar desarrollos de trastornos de la conducta alimentaria. Este hecho no solo incrementaría las dificultades en cuanto a la intervención y agravaría notablemente, no únicamente el bienestar psicológico del deportista, sino también su propia salud física en el caso de derivar en trastornos más severos de alimentación.

Finalmente, considerando tanto los datos de prevalencia como los resultados derivados de las diferencias de medias, se debe prestar especial atención al colectivo femenino de deportistas, ya que son estas quienes presentan una mayor tasa de prevalencia del síndrome y quienes, además, perciben de manera más negativa su esquema corporal, señalándolas así como un colectivo de alto riesgo.

Como conclusión, se puede afirmar que, tras la aplicación de un instrumento específico del 
contexto deportivo, los niveles de prevalencia del síndrome en España se sitúan en una tasa muy similar a la informada en otros países de ámbito internacional. Además, se confirma la relación hipotetizada por los autores entre la percepción del esquema corporal y el síndrome de burnout, destacando el peso existente respecto a la dimensión de la realización personal, la cual conlleva un componente psicológico notable.

Respecto a posibles limitaciones del estudio, puede destacarse el tipo de muestreo realizado, pues si bien se ha tratado de que sea representativo del panorama deportivo nacional, tanto a nivel geográfico como en función de las diferentes modalidades deportivas, idealmente sería necesario llevar a cabo un muestreo aleatorio, pero hay que tener en cuenta la dificultad que esto supone en un campo de trabajo tan amplio como el contexto deportivo.

De cara a futuras líneas de investigación, se plantea como necesaria la elaboración de un modelo de ecuaciones estructurales que implique un conjunto más amplio de variables psicológicas relacionadas con el síndrome de burnout. De este modo, será posible conocer el efecto que cada una de ellas presenta sobre el propio síndrome y reducir, mediante una intervención específica, tanto el riesgo de desarrollo del síndrome como posible alteraciones de la imagen corporal, así como en casos concretos, posibles alteraciones de la conducta alimentaria.

\section{Referencias}

Austria, F., Cruz, B., Herrera, L. \& Salas, J. (2012). Relaciones estructurales entre estrategias de afrontamiento y síndrome de burnout en personal de salud: un estudio de validez externa y de constructo. Universitas Psychologica, 11(1), 197-206.

Baum, A. (2006). Eating disorders in the male athlete. Sports Medicine, 36(1), 1-6.

Bernaldo de Quirós-Aragón, M. \& Labrador-Encinas, F. J. (2007). Evaluación del estrés laboral y burnout en los servicios de urgencia extrahospitalaria. International Journal of Clinical and Health Psychology, 7(2), 323-335.
Black, J. M. \& Smith, A. L. (2007). An examination of Coakley's perspective on identity, control, and burnout among adolescent athletes. International Journal of Sport Psychology, 38(4), 417-436.

Capel, S. A. (1986). Psychological and organizational factors related to burnout in athletic trainers. Research Quarterly for Exercise and Sport, 57(4), 321-328.

Carlin, M. \& de los Fayos Ruiz, E. (2010). El síndrome de burnout: evolución histórica desde el contexto laboral al ámbito deportivo. Anales de Psicología, 26(1), 169-180.

Conde, C., Sáenz-López, P., Carmona, J., González-Cutre, D., Martínez, C. \& Moreno, J. A. (2010). Validación del Cuestionario de Percepción de Soporte de la Autonomía en el Proceso de Entrenamiento (ASCQ) en jóvenes deportistas españoles. Estudios de Psicología, 31(2), 145-157.

Cratty, B. J. (1982). El desarrollo perceptivo y motor en los niños. Barcelona: Paidós.

Cresswell, S. L. \& Eklund, R. C. (2004). The athlete burnout syndrome: Proposed early signs. Journal of Science and Medicine in Sport, 7(4), 481-487.

Díaz, F., López, A. M. \& Varela, M. T. (2012). Factores asociados al síndrome de burnout en docentes de colegios de la ciudad de Cali, Colombia. Universitas Psychologica, 11(1), 217-227.

Etzion, D. (1984). Moderating effect of social support on the stress-burnout relationship. Journal of Applied Psychology, 69(4), 615-622.

Feigley, D. A. (1984). Psychological burnout in highlevel athletes. Physician and Sport Medicine, 12(10), 109-119.

Ferreira, C., Pinto-Gouveia, J. \& Duarte, C. (2011). The validation of the Body Image Acceptance and Action Questionnaire: Exploring the moderator effect of acceptance on disordered eating. International Journal of Psychology and Psychological Therapy, 11(3), 327-345.

Garcés de Los Fayos, E. J. (1999). Nivel de burnout y rendimiento deportivo. En E. Cantón (Ed.), Motivación y su aplicación al deporte (pp. 91-102). Valencia: Promolibro.

González, R., Molinero, O. \& Márquez, S. (2008). Un nuevo modelo integrador del sobreentrenamiento basado en las aproximaciones psicológicas del 
burnout y del estrés-recuperación. Ansiedad y Estrés, 14(2-3), 221-237.

Goodger, K., Gorely, T., Lavallee, D. \& Harwood, C. (2007). Burnout in sport: A systematic review. The Sport Psychologist, 21(2), 127-151.

Gustafsson, H. (2011). Are athletes burning out with passion? European Journal of Sport Science, 11(6), 387-395.

Gustafsson, H., Hassmeén, P., Kentta, G. \& Johansson, M. (2008). A qualitative analysis of burnout in elite Swedish athletes. Psychology of Sport and Exercise, 9(6), 800-816.

Gustafsson, H., Hassmeén, P. \& Podlog, L. (2010). Exploring the relationship between hope and burnout in competitive sport. Journal of Sports Sciences, 28(14), 1495-1504.

Gustafsson, H., Kenttä, G. \& Hassmén, P. (2011). Athlete burnout: An integrated model and future research directions. International Review of Sport and Exercise Psychology, 4(1), 3-24.

Gustafsson, H., Kenttä, G., Hassmén, P. \& Lundqvist, C. (2007). Prevalence of burnout in adolescent competitive athletes. The Sport Psychologist, 21(1), 21-37.

Gustafsson, H. \& Skoog, T. (2012). The mediational role of perceived stress in the relation between optimism and burnout in competitive athletes. Anxiety, Stress and Coping: An International Journal, 25(2), 183-199.

Holm-Denoma, J., Scaringi, V., Gordon, K., Van Orden, K. \& Joiner, T. (2009). Eating disorder symptoms among undergraduate varsity athletes, club athletes, independent exercisers, and nonexercisers. International Journal of Eating Disorders, 42(1), 47-53.

Jenaro-Río, C., Flores-Robaina, N. \& González-Gil, F. (2007). Síndrome de burnout y afrontamiento en trabajadores de acogimiento residencial de menores. International Journal of Clinical and Health Psychology, 7(1), 107-121.

León-Rubio, J. M., Cantero, F. J. \& León-Pérez, J. M. (2011). Diferencias del rol desempeñado por la autoeficacia en el burnout percibido por el personal universitario en función de las condiciones de trabajo. Anales de Psicología, 27(2), 518-526.

Lobera, I., Parra, S., Lopez, M. D., Galan, D., Casals, O. $\&$ Bautista, E. (2009). Screening for eating disor- ders in a psychiatric outpatient population. The European Journal of General Practice, 15(2), 107-111.

Murray, K., Byrne, D. \& Rieger, E. (2011). Investigating adolescent stress and body image. Journal of Adolescence, 34(2), 269-278.

Pedrosa, I. \& García-Cueto, E. (2010). Medidas psicométricas del síndrome de burnout en deportistas. Revista de Investigación para Alumnos de Psicología y Logopedia, 4(1), 45-68.

Pedrosa, I. \& García-Cueto, E. (2012). Adaptación al español del Athlete Burnout Questionnaire (ABQ) en una muestra española de deportistas. Ansiedad y Estrés, 18(2-3), 155-166.

Pedrosa, I., Martín-Vázquez, M. \& García-Cueto, E. (2008, marzo). Construcción de una escala de medida: intervención y comparaciones deportistas versus no deportistas. Trabajo presentado en el XI Congreso Nacional, XI Andaluz y III Iberoamericano de Psicología de la Actividad Física y del Deporte, Sevilla, España.

Pedrosa, I., Suárez-Álvarez, J. \& García-Cueto, E. (2012). Construcción de una Escala de valoración del Estrés en el Ámbito Deportivo (EEAD). Revista Electrónica de Metodología Aplicada, 17(2), 18-31.

Petrie, T., Greenleaf, C., Carter, J. \& Reel, J. (2007). Psychosocial correlates of disordered eating among male collegiate athletes. Journal of Clinical Sport Psychology, 1(4), 340-357.

Raedeke, T. D. (1997). Is burnout more than just stress? A sport commitment perspective. Journal of Sport and Exercise Psychology, 19(4), 396-417.

Raedeke, T. D. \& Smith, A. L. (2001). The Athlete Burnout Questionnaire Manual. Morgantown: West Virginia University, Fitness Information Technology.

Smith, R. E. (1986). Toward a cognitive-affective model of athletic burnout. Journal of Sport Psychology, $8(1), 36-50$.

Sundgot-Borgen, J. \& Torstveit, M. K. (2010). Aspects of disordered eating continuum in elite high-intensity sports. Scandinavian Journal of Medicine and Science in Sports, 20(2), 112-121.

Vives Benedicto, L. \& Garcés de los Fayos, E. (2004). Incidencia del síndrome de burnout en el perfil cognitivo en jóvenes deportistas de alto rendimiento. Cuadernos de Psicología del Deporte, 4(1-2), 29-43. 
